\title{
Genotoxicity Evaluation of an Ethanol Extract Mixture of Astragali Radix and Salviae miltiorrhizae Radix
}

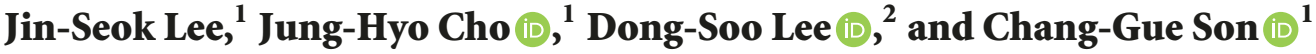 \\ ${ }^{1}$ Liver and Immunology Research Center, Oriental Medical Collage of Daejeon University, 22-5 Daehung-dong, \\ Jung-gu, Daejeon 301-724, Republic of Korea \\ ${ }^{2}$ Department of Internal Medicine, Daejeon St. Mary's Hospital of Catholic University, Daejeon, Republic of Korea
}

Correspondence should be addressed to Chang-Gue Son; ckson@dju.ac.kr

Received 26 July 2018; Accepted 2 October 2018; Published 9 October 2018

Academic Editor: Carlos H. G. Martins

Copyright (C) 2018 Jin-Seok Lee et al. This is an open access article distributed under the Creative Commons Attribution License, which permits unrestricted use, distribution, and reproduction in any medium, provided the original work is properly cited.

\begin{abstract}
Myelophil, a combination of Astragali Radix and Salviae Radix, is one of the most commonly used remedies for disorders of $Q i$ and blood in traditional Chinese medicine. Based on the clinical applications of these plants, in particular to pregnant woman, this study aimed to evaluate the genotoxic potential of an ethanol extract mixture of the above two herbs, called Myelophil. Following the Organization for Economic Cooperation and Development (OECD) Guideline methods, a genotoxicity test was conducted using a bacterial reverse mutation test with Salmonella typhimurium (TA98, TA100, TA1535, and TA1537) and Escherichia coli (WP2 $\mu \mathrm{vrA}$ ), an in vitro mammalian chromosome aberration test using a Chinese hamster ovary cell line (CHO-K1), and an in vivo mammalian erythrocyte micronucleus test using ICR mouse bone marrow. In the Ames test, for both types of mutations (base substitution and frameshift) under conditions with/without an S9 mix up to 5,000 $\mu \mathrm{g} /$ plate, Myelophil did not increase the number of revertant colonies of all $S$. typhimurium strains as well as E. coli strain. For both short $(6 \mathrm{~h})$ and long tests with/without $S 9$ mix, the chromosome aberration test did not show any significant increase in the number of structural or numerical chromosome aberrations by Myelophil. In addition, no significant change in the number of micronucleated polychromatic erythrocytes or polychromatic erythrocytes was observed in the bone marrow of an ICR mouse administered Myelophil orally at 2,000 mg/kg/day for 2 days, respectively. These results are the first to provide experimental evidence that Myelophil, an ethanol extract mixture of Astragali Radix and Salviae Radix, has no risk of genotoxicity.
\end{abstract}

\section{Introduction}

Herbal drugs have been used traditionally for various health benefits in East Asian countries, and they are now being adopted worldwide [1]. According to Global Industry Analysis, the global market for herbal medicinal products has grown continuously and was estimated at 107 billion US dollars in 2017. Herbal drugs are generally regarded as safe due to their derivation from natural resources and extensive clinical use for thousands of years [2]; however, recently many concerns have been raised regarding the adverse effects of herbal remedies and their low levels of quality control with standardization $[3,4]$.

In addition, the use of herbal products during pregnancy is common. A multinational study reported that $29.3 \%$ of pregnant women adopted herbal medicine [5]. The Swedish
Medical Birth Register study reported that 787 of 860,215 women $(0.9 \%)$ used herbal products during early pregnancy [6]. Accordingly, concerns about the potential risk for genotoxicity of herbal medicines and phytochemicals have arisen $[7,8]$. The Korean Ministry of Food and Drug Safety (MFDS) has developed a guideline (No. 2015-82, 2015) that requires genotoxicity testing prior to introducing any herb-derived new drug.

Myelophil is a $30 \%$ ethanolic extract consisting of equal parts of Astragali Radix and Salviae Radix that are representative herbs used to treat problems of Qi and blood in traditional Korean medicine [9]. Myelophil is commonly used to maintain Qi-blood homeostasis and to treat fatigueassociated disorders, even in children and pregnant women [10]. There are subchronic toxicity studies available for Astragali Radix and Myelophil [11, 12]; however, no genotoxicity 
evaluation of Myelophil, Astragali Radix, or Salviae Radix has been conducted to date.

To provide scientific evidence about the genotoxic risk of Myelophil, we herein conducted three genotoxicity tests, i.e., a bacterial reverse mutation test, a mammalian chromosome aberration test, and a mammalian erythrocyte micronucleus test.

\section{Materials and Methods}

2.1. Preparation of Myelophil and Fingerprinting Analysis. Myelophil contains a 30\% ethanolic extract of Astragali Radix (Astragalus membranaceus) and Salviae Radix (Salvia miltiorrhiza) in equal amounts. The Myelophil was manufactured by Kyung-Bang Pharmacy (Incheon, Rep. of Korea), according to the approved good manufacturing practice (GMP) guidelines of the Korean MFDS as previously reported [10]. Briefly, $100 \mathrm{~kg}$ of Myelophil was boiled in $1000 \mathrm{~L}$ of $30 \%$ ethanol for $4 \mathrm{~h}$ at $100^{\circ} \mathrm{C}$ and was then filtered using a 300 -mesh filter $(50 \mathrm{~mm})$. Some samples were filtered through filter paper (Advantec, Toyo Roshi Kaisha, Tokyo, Japan) and lyophilized for this study. The final Myelophil product [yield 20.52\% $(\mathrm{w} / \mathrm{w})]$ was stored for future use (VS No. KB-Myelo-1501).

In addition, for confirmation of the reproducibility of the Myelophil, fingerprinting analysis was conducted with six reference compounds: astragaloside IV (SMB003158) and formononetin (94334) for Astragali Radix, and salvianolic acid A (97599), B (SML0048), C (APC-657), D (APC-658), and rosmarinic acid (R4033) for Salviae Radix (Supplementary Figure 1). Each reference compound was purchased from Sigma (St. Louis, St. Louis, MO, USA) or Aktin Chemical, Inc., (Chengdu, China). Briefly, $1 \mathrm{mg}$ of Myelophil and $10 \mu \mathrm{g}$ of each reference compound were prepared in $90 \%$ methanol, and then filtrates were analysed using ultra-high-performance liquid chromatography (UHPLC, Thermo Scientific, San Jose, CA, USA) coupled with high resolution LTQ Orbitrap mass spectrometry (MS) system (Thermo Scientific Co., San Jose, CA, USA).

2.2. Bacterial Reverse Mutation Test (Ames Test). The bacterial reverse mutation test (Test \# GT17-195) was conducted by a good-laboratory-practice- (GLP-) proven laboratory (Korean Conformity Laboratories, Incheon, Rep. of Korea) in accordance with OECD guideline No. 471 (Adapted 21th July 1997). Four histidine-requiring Salmonella typhimurium strains, TA98, TA100, TA1535, and TA1537, and one tryptophan-requiring Escherichia coli strain, WP2 $\mu \mathrm{vrA}$, were obtained from Molecular Toxicology Inc. (Boone, NC, USA). The positive controls were sodium azide (NaN3), 9aminoacridine hydrochloride hydrate (9-AA), 2-(2-furyl)-3(5-nitro-2-furyl) acrylamide (AF-2), and 2-aminoanthracene (2-AA), respectively. According to the results of the preliminary dose-range test, 5,000 $\mu \mathrm{g} /$ plate was selected as the highest concentration followed by $1667,556,185$, and $62 \mu \mathrm{g} /$ plate in the present study. The present study was tested using triplicates for each dose for each bacterial strain with or without the S9 mix. Briefly, we added $0.05 \mathrm{ml}$ of Myelophil, distilled water (negative control) or positive control (AF-2 0.01 or $0.1 \mu \mathrm{g} /$ plate, NaN3 $0.5 \mu \mathrm{g} /$ plate, 9 -AA $80 \mu \mathrm{g} /$ plate or 2-AA
0.5 to $10 \mu \mathrm{g} /$ plate) to $2.0 \mathrm{ml}$ of top agar (held at $45^{\circ} \mathrm{C}$ ) along with a $0.1 \mathrm{ml}$ precultured tester strain and $0.5 \mathrm{ml}$ of either the S9 mix or $0.2 \mathrm{M}$ phosphate buffer ( $\mathrm{pH} 7.4$ ). The mixture was vortexed then poured onto a minimal medium agar plate. After the agar overlay solidified, the plates were inverted and incubated at $37^{\circ} \mathrm{C}$ for $48 \mathrm{~h}$. Following incubation, we manually counted the revertant colonies.

2.3. In Vitro Mammalian Chromosome Aberration Test. The chromosome aberration test (Test \# GT17-196) was conducted by a GLP-proven laboratory (Korean Conformity Laboratories, Incheon, Rep. of Korea) in accordance with OECD guideline No. 473 (Adapted 29th July 2016). The Chinese hamster ovary cell line (CHO-K1) was obtained from the Korean Cell Line Bank (KCLB, Seoul, Korea) and cultured in minimum essential medium (Gibco, California, USA) supplemented with $10 \%$ foetal bovine serum (Gibco) at $37^{\circ} \mathrm{C}$ and $5 \% \mathrm{CO}_{2}$. To determine the maximum concentration for the present study, the proliferation assay was conducted up to a dose of 5,000 $\mu \mathrm{g} / \mathrm{mL}$ with or without the $\mathrm{S} 9 \mathrm{mix}$. Based on the results from the proliferation assay, the present study was designed to apply Myelophil to $\mathrm{CHO}-\mathrm{K} 1$ cells without the S9 mix (up to $185.19 \mu \mathrm{g} / \mathrm{mL}$ for $24 \mathrm{~h}$, and $6 \mathrm{~h}$ followed by 18 $\mathrm{h}$ for recovery) or with the S9 mix (up to $555.56 \mu \mathrm{g} / \mathrm{mL}$ for $6 \mathrm{~h}$ followed by $18 \mathrm{~h}$ for recovery), respectively. Mitomycin C (MMC, $0.04 \mu \mathrm{g} / \mathrm{mL}$ ) or cyclophosphamide monohydrate $(\mathrm{CPA}, 10 \mu \mathrm{g} / \mathrm{mL}$ ) was used as a positive control in the $\mathrm{S} 9 \mathrm{mix}$ absent or S9 mix present conditions, respectively. Following incubation and staining with 5\% Giemsa (Merck, Germany, \# HX69072204), we examined the structural and numerical chromosomal aberrations under an optical microscope.

2.4. In Vivo Mammalian Erythrocyte Micronucleus Test. The in vivo mammalian erythrocyte micronucleus test (Test \# GT17-197) was conducted by a GLP-proven laboratory (Korean Conformity Laboratories, Incheon, Rep. of Korea) in accordance with OECD guideline No. 474 (Adopted July 21, 2016). Specific pathogen-free male (37) and female (12) ICR mice (7 weeks old, 30-32 g) were obtained from Orient Co., Ltd. (Seongnam, Korea). After one-week acclimatization, a dose-finding test was conducted using each of 12 male and female mice given Myelophil (each 3 mice for three dose groups of 500,1000 , or $2000 \mathrm{mg} / \mathrm{kg}$ bw/day, orally once a day) for 4 days. Based on no treatment-related clinical signs and no differences between males and females, Myelophil was administered once daily for two days by gavage to 25 male mice (each 5 mice for $0,500,1000,2000 \mathrm{mg} / \mathrm{kg}$ and a positive control) for the micronucleus test. MMC (2 mg/kg bw/day in normal saline, only once on the final day of drug administration) was administered by intraperitoneal injection as a positive control. Mice were sacrificed by $\mathrm{CO}_{2}$ gas inhalation at $24 \mathrm{~h}$ after the last administration and their bone marrow cells were prepared as described [13]. We determined the proportion of immature erythrocytes (polychromatic erythrocytes, PCE) to total erythrocytes (immature plus mature erythrocytes, normochromatic erythrocytes, NCE) for each animal by analysing at least 500 erythrocytes. In addition, a minimum of 4,000 PCE was scored for the incidence of micronucleated polychromatic erythrocytes (MNPCE). 


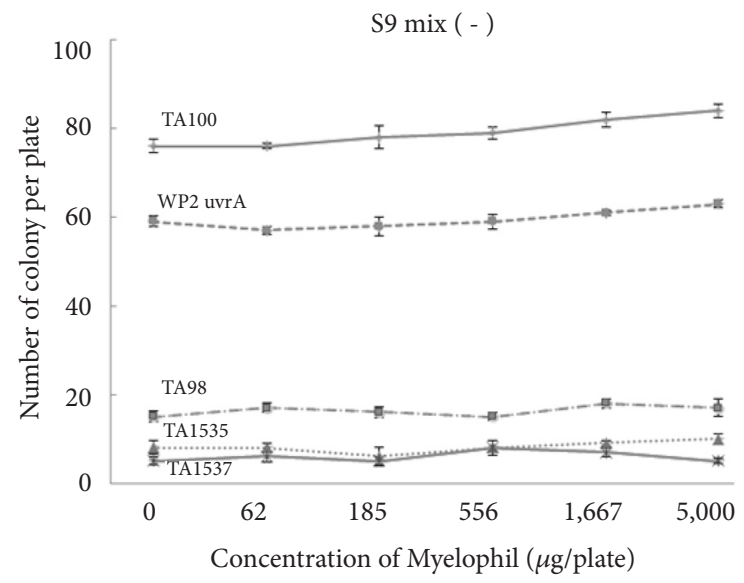

(a)

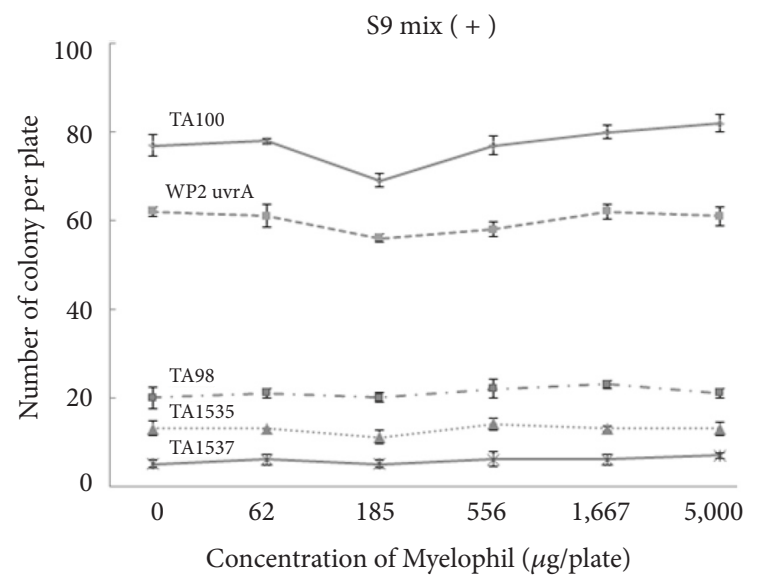

(b)

\begin{tabular}{|c|c|c|c|c|c|c|}
\hline \multirow{2}{*}{ Condition } & \multirow{2}{*}{ Strain } & \multicolumn{3}{|c|}{ Base-pair substitution type } & \multicolumn{2}{|c|}{ Frameshift type } \\
\hline & & TA100 & TA1535 & WP2uvrA & TA98 & TA1537 \\
\hline \multirow{2}{*}{ S9 mix ( - ) } & Positive control (dose) & AF-2 (0.01) & $\mathrm{NaN}_{3}(0.5)$ & AF-2 (0.01) & AF-2 (0.1) & 9-AA (80) \\
\hline & N. of colony & $562 \pm 80.7$ & $277 \pm 19.6$ & $348 \pm 18.3$ & $314 \pm 26.0$ & $1,976 \pm 37.2$ \\
\hline \multirow{2}{*}{ S9 mix (+ ) } & Positive control (dose) & $2-\mathrm{AA}(1.0)$ & $2-\mathrm{AA}(2.0)$ & 2-AA (10) & $2-\mathrm{AA}(0.5)$ & 2-AA (2.0) \\
\hline & N. of colony & $475 \pm 47.8$ & $329 \pm 3.1$ & $374 \pm 22.8$ & $359 \pm 7.1$ & $314 \pm 2.6$ \\
\hline
\end{tabular}

(c)

FIGURE 1: Bacterial reverse mutation test: five bacterial strains were treated with Myelophil in the absence (a) or presence (b) of S9 mix. The significant increase of revertant colonies by positive control verified the adequate condition of method used (c).

This study was reviewed and assessed by the Institutional Animal Care and Use Committee (IACUC) of the Korean Institute of Toxicology (IA17-00520). All animals were cared for in accordance with the principles outlined in the National Institutes of Health (NIH) Guide for the Care and Use of Laboratory Animals.

2.5. Statistical Analysis. No statistical analysis was conducted on the Ames test results. Instead, the presence of at least dose-dependent one strain or reproducible increased colony pattern at over a certain concentration indicated a positive result. The statistical analyses for the in vitro chromosome aberration test were performed as described previously [14]. The number of aberrant metaphases and the number of [polyploidy (PP, $\geq 37$ chromosomes) + endoreduplication (ER)] were analysed by comparing them to the negative and positive controls using an $\chi 2$ test and Fisher's exact test. In vivo micronucleus results were evaluated according to the minor-modified methods by Lovell's study [15], and the statistical analysis was performed as previously described [16]. Statistical analyses were conducted using the SPSS 12.0 $\mathrm{K}$ program, and the differences were regarded as significant at $\mathrm{P}<0.05$.

\section{Results}

3.1. Bacterial Reverse Mutation Test (Ames Test). Myelophil did not show any cytotoxicity in Salmonella typhimurium
(TA98, TA100, TA1535, and TA1537) or Escherichia coli (WP2uvrA) at up to $5,000 \mu \mathrm{g} /$ plate. The positive control compounds significantly increased the numbers of revertant colonies by more than 5 folds compared to the negative control, confirming the sensitivity of the test system (Figure 1(c)). No biologically relevant increase in revertant colonies following treatment with Myelophil at up to 5,000 $\mu \mathrm{g} /$ plate was observed in any of the five tester strains in the absence or presence of $S 9$ metabolic activation (Figures 1(a) and 1(b)).

3.2. Chromosome Aberration Test. Based on the cell proliferation inhibition assay, the maximum concentration of Myelophil was determined to be $185.19 \mu \mathrm{g} / \mathrm{mL}$ and 555.56 $\mu \mathrm{g} / \mathrm{mL}$ in the absence and presence of the $\mathrm{S} 9 \mathrm{mix}$, respectively. Significant increase in frequency of chromosome aberration (per 100 metaphase cells) was observed in positive control (mitomycin C and cyclophosphamide monohydrate) compared to the negative control $(\mathrm{p}<0.01)$. In contrast, there is no significant increase in aberrant metaphase including structural or numerical aberrations at any dose of Myelophil with or without S9 metabolic activation (Table 1).

3.3. Micronucleus Test. There was no significant change in body weight between the first and final administration in the Myelophil or mitomycin C groups. The frequency of MNPCE per 4,000 PCE significantly increased in the mitomycin C 
TABLE 1: Chromosomal aberrations by Myelophil with and without $S 9$ activation.

\begin{tabular}{|c|c|c|c|c|c|c|}
\hline \multirow{2}{*}{ Condition } & \multirow{2}{*}{ Dose $(\mu \mathrm{g} / \mathrm{mL})$} & \multicolumn{4}{|c|}{ Chromosome aberrations ( $\mathrm{N}$. in 300 cells) } & \multirow{2}{*}{ Aberration rate (\%) } \\
\hline & & Break & Exchange & Numerical & Sum & \\
\hline \multirow{5}{*}{$\begin{array}{l}0-24 \mathrm{~h} \\
\text { S9 mix(-) }\end{array}$} & 0 & 1 & 0 & 0 & 1 & 0.33 \\
\hline & 20.68 & 2 & 0 & 0 & 2 & 0.67 \\
\hline & 61.73 & 1 & 1 & 0 & 2 & 0.67 \\
\hline & 185.19 & 2 & 0 & 0 & 2 & 0.67 \\
\hline & $\operatorname{MC}(0.04) * *$ & 9 & 64 & 0 & 73 & 24.33 \\
\hline \multirow{5}{*}{$\begin{array}{l}6-18 \mathrm{~h} \\
\text { S9 mix(-) }\end{array}$} & 0 & 1 & 0 & 0 & 1 & 0.33 \\
\hline & 20.68 & 0 & 1 & 0 & 1 & 0.33 \\
\hline & 61.73 & 2 & 1 & 0 & 3 & 1.00 \\
\hline & 185.19 & 2 & 1 & 0 & 3 & 1.00 \\
\hline & MC & 14 & 56 & 0 & 70 & 23.33 \\
\hline \multirow{5}{*}{$\begin{array}{l}6-18 \mathrm{~h} \\
\text { S9 mix (+) }\end{array}$} & 0 & 1 & 1 & 0 & 2 & 0.67 \\
\hline & 61.73 & 0 & 2 & 0 & 2 & 0.67 \\
\hline & 185.19 & 1 & 1 & 0 & 2 & 0.67 \\
\hline & 555.56 & 2 & 0 & 0 & 2 & 0.67 \\
\hline & CPA $(10) * *$ & 10 & 62 & 0 & 72 & 24.00 \\
\hline
\end{tabular}

Structural (chromosomal break or exchange) and numerical aberration (polyploidy or endoreduplication) were counted in 300 CHO-K1 cells. MC: mitomycin C; CPA: cyclophosphamide. $* * \mathrm{p}<0.01$ vs. negative control.

TABLE 2: Bone marrow micronucleus assay results of male mice.

\begin{tabular}{lcccc}
\hline $\begin{array}{l}\text { Dose } \\
(\mathrm{mg} / \mathrm{kg} \text { bw/day })\end{array}$ & \multicolumn{2}{c}{ Body weight $(\mathrm{g})$} & $\begin{array}{c}\text { MNPCE/4000 PCEs(Mean } \\
\pm \text { SD, \%) }\end{array}$ & PCE/(PCE+NCE) \\
\hline 0 & day 0 & day 2 & $0.19 \pm 0.09$ & $0.57 \pm 0.01$ \\
500 & $34.3 \pm 1.4$ & $34.2 \pm 4.5$ & $0.20 \pm 0.07$ & $0.56 \pm 0.01$ \\
1000 & $35.1 \pm 1.4$ & $35.7 \pm 1.3$ & $0.20 \pm 0.08$ & $0.54 \pm 0.05$ \\
2000 & $35.7 \pm 1.2$ & $35.7 \pm 1.3$ & $0.23 \pm 0.06$ & $0.57 \pm 0.02$ \\
MC $(2.0) * *$ & $34.8 \pm 0.7$ & $35.7 \pm 0.8$ & $9.59 \pm 1.74$ & $0.41 \pm 0.05$ \\
\hline
\end{tabular}

Micronucleus assay was conducted in ICR mice after oral administration for 2 days, and then the number of MNPCE (micronucleated polychromatic erythrocytes) in 4,000 PCE (polychromatic erythrocyte) was analysed. The proportion of PCE and NCE (normochromatic erythrocytes) in 500 erythrocytes was determined. MC: mitomycin C. $* * \mathrm{p}<0.01 v s$. negative control.

group ( $\mathrm{p}<0.01$ ); however, no dose in the Myelophil group showed a change in the MNPCE incidence compared to the negative control. In addition, there was no significant decrease in the $\mathrm{PCE} /(\mathrm{PCE}+\mathrm{NCE})$ compared to the negative control (Table 2).

\section{Discussion}

A genotoxicity study is a key step for risk assessment during drug development for protecting human health because various genotoxic compounds can cause a DNA damage such as cross-links, adducts, and cleavage [17]. Eventually genotoxins can lead to tumor progression [18] and congenital malformations [19]. Recently, some issues regarding herb-related genotoxicity have arisen. In particular, there are concerns about the frequent exposure of pregnant women and their foetuses to phytomedicines and herbal supplements without adequate knowledge of the safety during pregnancy [8]. An analysis of 14,551 births reported an increased risk of congenital malformations of the nervous system after exposure during the first trimester of pregnancy to Rhizoma Coptidis, a typical medicinal herb with the property of "clearing dampheat" [20].

In this study, we conducted a genotoxic risk assessment of Myelophil, an ethanolic extract combining Astragali Radix and Salviae Radix. We employed a bacterial reverse mutation test, a mammalian chromosome aberration test, and a mammalian erythrocyte micronucleus test. One recent review article summarized the genotoxicity of several Chinese medicinal plants, and methanol extracts of Elephantopus Scaber leaf exhibited the abnormalities in both mitotic index and chromosome aberrations [21]. As expected, the positive control agents significantly induced genotoxicity, which validated the tests used. However, no genotoxic positive result was observed in Myelophil treated groups compared to vehicle control (Figure 1, Tables 1 and 2). These results indicate that Myelophil does not exhibit any genotoxic risk under the experimental conditions of this study. The above tests have been used internationally as the minimal three-test battery by the International Council for Harmonisation of Technical 
Requirements for Pharmaceuticals for Human Use (ICH) since 1997 [22].

Myelophil has been prescribed to a wide spectrum of patients complaining of chronic fatigue and bone marrow dysfunctions in Daejeon University Hospitals since 2002. Astragali Radix and Salviae Radix, the two components of Myelophil, are very commonly used as medicinal herbs. Astragali Radix has pharmacological properties such as antiinflammatory or immunomodulatory effects [23, 24], whereas Salviae Radix exhibited antioxidant and cardioprotective properties $[25,26]$, respectively. There are many active compounds, including astragaloside IV, tanshinones, and salvianolic acids, known to be present in these two herbs [2729]. There have been general toxicological studies for Astragali Radix, Salviae Radix, and their combination conducted previously [11, 30]. However, our current study is the first genotoxic risk assessment for Astragali Radix and Salviae Radix.

The three-test battery applied in our study has limitations, especially the use of bacterial test organisms; however, its practicality is well validated. In fact, the Rhizoma-Coptidisrelated risk of congenital malformations is supported by the genotoxicity testing of Hwanglyeonhaedok-tang. Rhizoma Coptidis is the main herb in this formula, and revertant colonies and chromosome aberrations were observed in these tests [31]. For compounds giving negative results like Myelophil, the completion of this 3-test battery usually provides a sufficient level of safety to demonstrate the absence of genotoxic activity [32].

Taken together, it was found that Myelophil is neither mutagenic in the in vitro systems nor clastogenic in the in vivo system. Based on the above results, we can conclude that Myelophil poses no genotoxic risks. Additionally, this study will provide important information for the use of Astragali Radix and Salviae Radix as medicinal resources in the future.

\section{Data Availability}

The data used to support the findings of this study are available from the corresponding author upon request.

\section{Additional Points}

This manuscript has been checked by at least two native speakers of English in scientific and technical editing service company (Nature Publishing Group language editing). For a certificate, please see languageediting.nature.com/certificate (Reference number:19XRCXLS-413B-2D67-E849-C20F-E569).

\section{Conflicts of Interest}

The authors declare that no conflicts of interest exist. All the authors have approved the final article.

\section{Authors' Contributions}

Jin-Seok Lee (neptune26ljs@naver.com) wrote the main manuscript text and conducted experiments. Jung-Hyo
Cho (choajoa@dju.kr) prepared Figure 1 and tables. DongSoo Lee (cmcdj9502@catholic.ac.kr) supported the statistical analysis and technical experiments. Chang-Gue Son (ckson@dju.ac.kr) supervised the manuscript and directed final version of all contents. All authors reviewed and approved this manuscript.

\section{Acknowledgments}

This research was supported by the National Research Foundation of Korea (NRF) funded by the Ministry of Education, Science and Technology (NRF-2018R1A6A1A03025221) and by the Oriental Medicine R\&D Project, Ministry of Health \& Welfare, Republic of Korea (HI15C0112).

\section{Supplementary Materials}

For analysis, ultra-high-performance liquid chromatography (UHPLC) coupled with high resolution LTQ Orbitrap mass spectrometry (MS) system was used for fingerprinting analysis of Myelophil. The UHPLC separation was performed on an Accela UHPLC system using an Acquity BEH C18 column $(1.7 \mu \mathrm{m}, 100 \times 2.1 \mathrm{~mm}$; Waters $)$. The column was eluted at a flow rate of $0.3 \mathrm{~mL} / \mathrm{min}$ using water $/ 0.1 \%$ formic acid and acetonitrile $/ 0.1 \%$ formic acid as mobile phases $A$ and $\mathrm{B}$, respectively, with the following gradients: $0-1 \mathrm{~min}, 10 \% \mathrm{~B}$ (isocratic); 1-10 min, 10-90\% B (linear gradient); and 10-12 min, 100\% B (isocratic). (Supplementary Materials)

\section{References}

[1] J. T. Coon and E. Ernst, "Panax ginseng: a systematic review of adverse effects and drug interactions," Drug Safety, vol. 25, no. 5, pp. 323-344, 2002.

[2] S. A. Jordan, D. G. Cunningham, and R. J. Marles, "Assessment of herbal medicinal products: challenges, and opportunities to increase the knowledge base for safety assessment," Toxicology and Applied Pharmacology, vol. 243, no. 2, pp. 198-216, 2010.

[3] P. Posadzki, L. K. Watson, and E. Ernst, "Adverse effects of herbal medicines: an overview of systematic reviews," Clinical Medicine, vol. 13, no. 1, pp. 7-12, 2013.

[4] J. Zhang, B. Wider, H. Shang, X. Li, and E. Ernst, "Quality of herbal medicines: challenges and solutions," Complementary Therapies in Medicine, vol. 20, no. 1-2, pp. 100-106, 2012.

[5] D. A. Kennedy, A. Lupattelli, G. Koren, and H. Nordeng, "Safety classification of herbal medicines used in pregnancy in a multinational study," BMC Complementary and Alternative Medicine, vol. 16, no. 1, 2016.

[6] L. Holst, H. Nordeng, and S. Haavik, "Use of herbal drugs during early pregnancy in relation to maternal characteristics and pregnancy outcome," Pharmacoepidemiology and Drug Safety, vol. 17, no. 2, pp. 151-159, 2008.

[7] J. Zhou, M. Ouedraogo, F. Qu, and P. Duez, "Potential genotoxicity of traditional Chinese medicinal plants and phytochemicals: An overview," Phytotherapy Research, vol. 27, no. 12, pp. 1745-1755, 2013.

[8] L. O. Bruno, R. S. Simoes, M. de Jesus Simoes, M. J. Girão, and O. Grundmann, "Pregnancy and herbal medicines: An unnecessary risk for women's health-A narrative review," Phytotherapy Research, vol. 32, no. 5, pp. 796-810, 2018. 
[9] J. H. Cho, C. K. Cho, J. W. Shin, J. Y. Son, W. Kang, and C. G. Son, "Myelophil, an extract mix of Astragali Radix and Salviae Radix, ameliorates chronic fatigue: a randomised, double-blind, controlled pilot study," Complementary Therapies in Medicine, vol. 17, no. 3, pp. 141-146, 2009.

[10] H.-G. Kim, J.-S. Lee, M.-K. Choi, J.-M. Han, and C.-G. Son, "Ethanolic extract of astragali radix and salviae radix prohibits oxidative brain injury by psycho-emotional stress in whisker removal rat model," PLoS ONE, vol. 9, no. 5, 2014.

[11] S.-Y. Yu, H.-T. OuYang, J.-Y. Yang et al., "Subchronic toxicity studies of Radix Astragali extract in rats and dogs," Journal of Ethnopharmacology, vol. 110, no. 2, pp. 352-355, 2007.

[12] J.M.S. J. W. Jung, J. Y. Son, N. W. Seong et al., "Four-week repeated dose toxicity test for myelophil in SD rats," Journal of Korean Medicine, vol. 30, no. 3, pp. 79-85, 2009.

[13] W. Schmid, “The micronucleus test," Mutation Research, vol. 31, no. 1, pp. 9-15, 1975.

[14] C. W. D. A. Richardson, J. A. Allen, G. Amphlett, D. O. Chanter, and B. Phillips, "Analysis of data from in vitro cytogenetic assays," in Statistical Evaluation of Mutagenicity Test Data, D. J. Kirkland, Ed., pp. 141-154, Cambridge University Press, 1989.

[15] D. D. P. A. Lovell, R. Albanese, G. E. Amphlett et al., "Statistical analysis of in vivo cytogenetic assays," in Statistical Evaluation Of Mutagenicity Test Data, D. J. Kirkland, Ed., pp. 184-232, Cambridge University Press, Cambridge, UK, 1989.

[16] M. Lee, C. Seo, J. Kim, and H. Shin, "Genotoxicity evaluation of Oryeong-san water extract using in vitro and in vivo tests," $B M C$ Complementary and Alternative Medicine, vol. 15, no. 1, 2015.

[17] G. M. Williams, "Mechanisms of chemical carcinogenesis and application to human cancer risk assessment," Toxicology, vol. 166, no. 1-2, pp. 3-10, 2001.

[18] S. J. Lee, Y. N. Yum, S. C. Kim et al., "Distinguishing between genotoxic and non-genotoxic hepatocarcinogens by gene expression profiling and bioinformatic pathway analysis," Scientific Reports, vol. 3, no. 1, 2013.

[19] R. Grazuleviciene, V. Kapustinskiene, J. Vencloviene, J. Buinauskiene, and M. J. Nieuwenhuijsen, "Risk of congenital anomalies in relation to the uptake of trihalomethane from drinking water during pregnancy," Occupational and Environmental Medicine, vol. 70, no. 4, pp. 274-282, 2013.

[20] C.-H. Chuang, P. Doyle, J.-D. Wang, P.-J. Chang, J.-N. Lai, and P.-C. Chen, "Herbal medicines used during the first trimester and major congenital malformations: An analysis of data from a pregnancy cohort study," Drug Safety, vol. 29, no. 6, pp. 539$548,2006$.

[21] K. Y. Ping, S. Shohaimi, S. Sasidharan, and U. K. Yushf, "Genotoxicity of selected chinese medicinal plants, elephantopus scaber, glycyrrhiza uralensis and salvia miltiorrhiza on allium cepa assay," Annals of Pharmacology and Pharmaceutics, vol. 2, no. 13, pp. 1070-1075, 2017.

[22] E. Lorge, V. Gervais, N. Becourt-Lhote, C. Maisonneuve, J.-L. Delongeas, and N. Claude, "Genetic toxicity assessment: Employing the best science for human safety evaluation part IV: A strategy in genotoxicity testing in drug development: Some examples," Toxicological Sciences, vol. 98, no. 1, pp. 39-42, 2007.

[23] M. Ryu, E. H. Kim, M. Chun et al., "Astragali Radix elicits anti-inflammation via activation of MKP-1, concomitant with attenuation of p38 and Erk," Journal of Ethnopharmacology, vol. 115, no. 2, pp. 184-193, 2008.

[24] M.-C. Kim, G.-H. Lee, S.-J. Kim et al., "Immune-enhancing effect of Danggwibohyeoltang, an extract from Astragali Radix and Angelicae gigantis Radix, in vitro and in vivo," Immunopharmacology and Immunotoxicology, vol. 34, no. 1, pp. 66-73, 2012.

[25] Y.-C. Hung, P.-W. Wang, T.-L. Pan, G. Bazylak, and Y.-L. Leu, "Proteomic screening of antioxidant effects exhibited by Radix Salvia miltiorrhiza aqueous extract in cultured rat aortic smooth muscle cells under homocysteine treatment," Journal of Ethnopharmacology, vol. 124, no. 3, pp. 463-474, 2009.

[26] L. Wang, Y. Li, W. Deng et al., "Cardio-protection of ultrafine granular powder for Salvia miltiorrhiza Bunge against myocardial infarction," Journal of Ethnopharmacology, vol. 222, pp. 99106,2018

[27] D.-Q. Zhang, J.-S. Li, Y.-M. Zhang, F. Gao, and R.-Z. Dai, “Astragaloside IV inhibits Angiotensin II-stimulated proliferation of rat vascular smooth muscle cells via the regulation of CDK2 activity," Life Sciences, vol. 200, pp. 105-109, 2018.

[28] S. Ma, D. Zhang, H. Lou, L. Sun, and J. Ji, "Evaluation of the anti-inflammatory activities of tanshinones isolated from Salvia miltiorrhiza var. Alba roots in THP-1 macrophages," Journal of Ethnopharmacology, vol. 188, pp. 193-199, 2016.

[29] T. Yu, P. Paudel, S. H. Seong, J. A. Kim, H. A. Jung, and J. S. Choi, "Computational insights into $\beta$-site amyloid precursor protein enzyme 1 (BACE1) inhibition by tanshinones and salvianolic acids from Salvia miltiorrhiza via molecular docking simulations," Computational Biology and Chemistry, vol. 74, pp. 273-285, 2018.

[30] M. Wang, J. Liu, B. Zhou et al., "Acute and sub-chronic toxicity studies of Danshen injection in Sprague-Dawley rats," Journal of Ethnopharmacology, vol. 141, no. 1, pp. 96-103, 2012.

[31] S. E. Jin, M.-Y. Lee, C.-S. Seo, H. Ha, J.-Y. Kim, and H.-K. Shin, "Genotoxicity evaluation of Hwanglyeonhaedok-tang, an herbal formula," Journal of Ethnopharmacology, vol. 202, pp. 122-126, 2017.

[32] I.C.o.H. (ICH), International Conference on Harmonization of Technical Requirements for Registration of Pharmaceuticals for Human Use. ICH Tripartite Guideline S2B. Genotoxicity: A Standard Battery for Genotoxicity Testing of Pharmaceuticals, European Federation of Pharmaceutical Industries' Associations Brussels, 2011. 


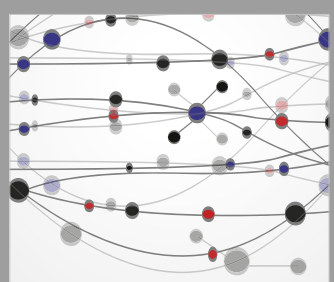

The Scientific World Journal
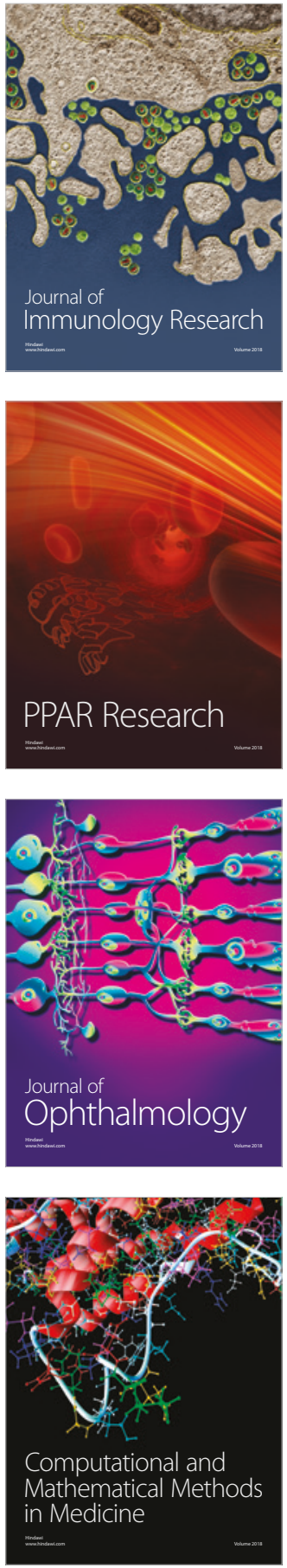

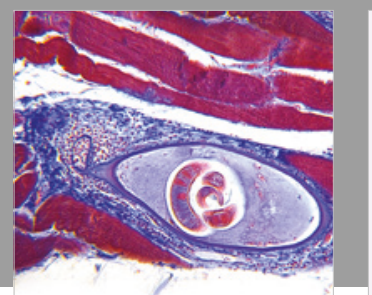

Gastroenterology Research and Practice

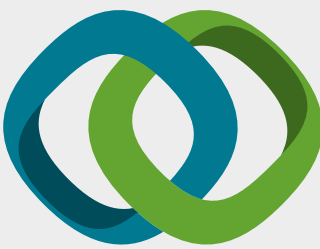

\section{Hindawi}

Submit your manuscripts at

www.hindawi.com
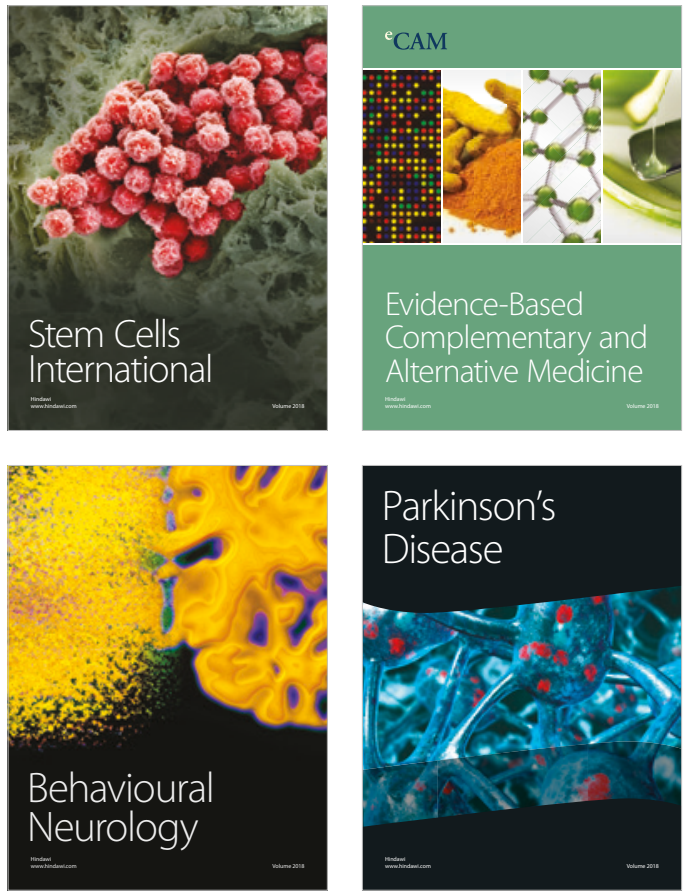

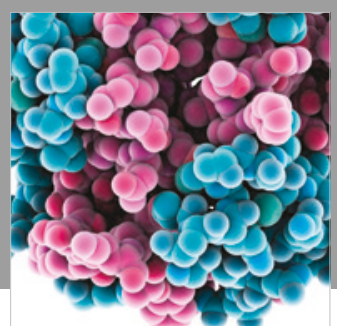

ournal of

Diabetes Research

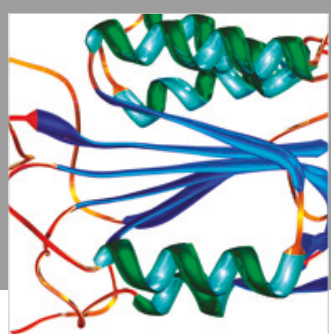

Disease Markers
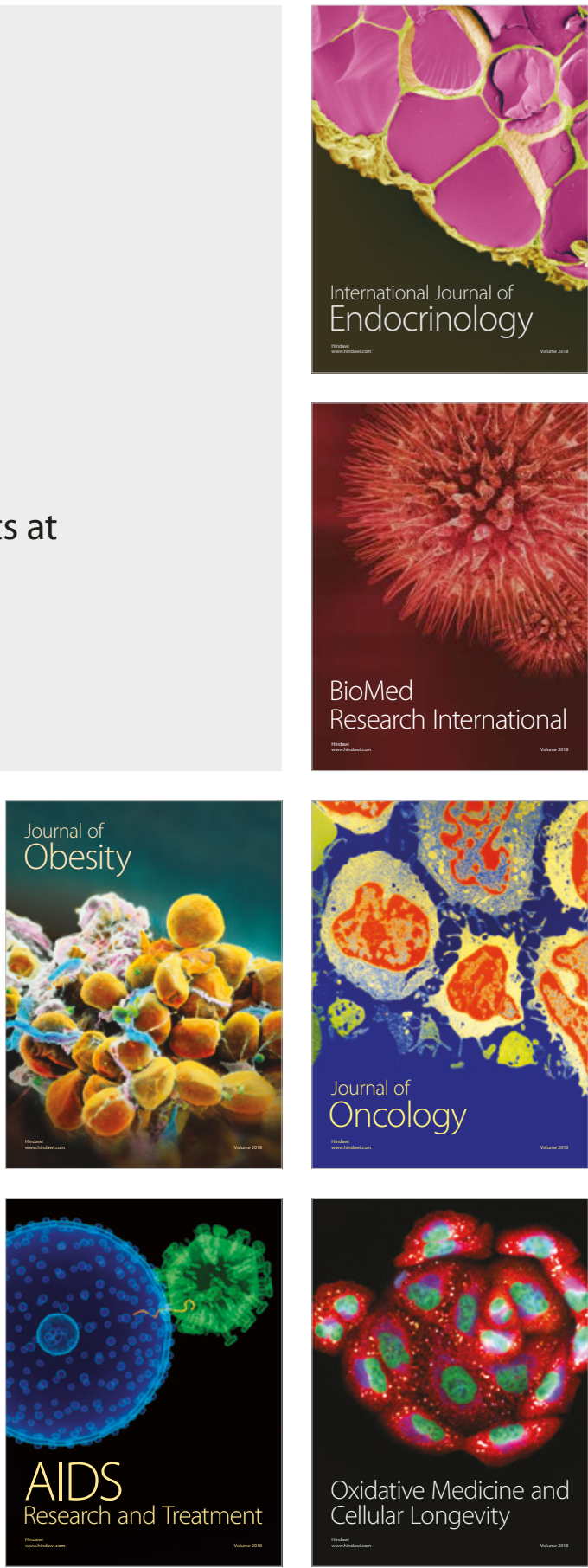